\title{
Elevated plasma succinate in PTEN, SDHB, and SDHD mutation-positive individuals
}

\author{
Judith A. Hobert, PhD'1, Jessica L. Mester, MS, CGC ${ }^{1,2}$, Jessica Moline, MS, CGC $^{1,2}$ and \\ Charis Eng, MD, $\mathrm{PhD}^{1-4}$
}

Purpose: Cowden syndrome results from germline mutations in the gene for phosphatase and tensin homologue deleted on chromosome $10($ PTEN) and from variants in succinate dehydrogenase B and D subunits. We hypothesized that succinate accumulation may be common among individuals with $S D H$ variants/mutations and those with PTEN mutations.

Methods: Urine and blood were collected from individuals meeting full or partial Cowden syndrome diagnostic criteria or those with paraganglioma (PGL) or a known susceptibility paragangliomaassociated gene mutation, and succinate was measured. PTEN, SDHB, $S D H C$, and $S D H D$ genes were sequenced from genomic DNA.

Results: Elevated plasma succinate was observed in 13/21 (62\%) individuals with germline PTEN, $S D H B$, or $S D H D$ mutations as compared with $5 / 32(16 \%)$ controls $(P<0.001)$, in $10 / 15(67 \%)$ individu- als with pathogenic PTEN mutations but in $<20 \%$ of mutation-negative individuals meeting identical criteria, and in individuals with mutations in SDHB $(1 / 1,100 \%)$ and SDHD $(2 / 5,40 \%)$.

Conclusion: Our data suggest that mutations in PTEN, SDHB, and $S D H D$ reduce catalytic activity of succinate dehydrogenase, resulting in succinate accumulation, and identify a common biochemical alteration in these two patient populations (PTEN and SDHx mutation positive individuals). Plasma organic acid analysis may provide an effective and inexpensive screening method to determine when more expensive gene sequencing of PTEN and SDH genes is warranted.

Genet Med 2012:14(6):616-619

Keywords: Cowden syndrome; PTEN; SDH; succinate syndrome

\section{INTRODUCTION}

Cowden syndrome (CS, OMIM\# 158350) is an underdiagnosed, difficult-to-recognize (affecting 1 in 200,000 individuals), autosomal dominant cancer syndrome with high penetrance. It is primarily associated with an increased risk of breast, follicular thyroid, and endometrial cancers; however, papillary thyroid cancer and renal cell carcinoma have also been reported. ${ }^{1-3}$ Clinical diagnosis of CS is made when an individual meets diagnostic criteria, a combination of pathognomonic major and/or minor criteria, established by the International Cowden Consortium. ${ }^{1}$ Many individuals meet partial diagnostic criteria for CS (defined as full criteria minus one) and are referred to as "CS-like." Germline mutations or deletions in phosphatase and tensin homologue deleted on chromosome 10 (PTEN, OMIM +601728), a ubiquitously expressed tumor suppressor, have been identified in $\sim 25 \%$ of individuals with CS, and somatic $P T E N$ mutations have been variably observed in a large number of sporadic malignancies. ${ }^{2,4}$

Germline heterozygous mutations in succinate dehydrogenase $(\mathrm{SDH}) \mathrm{B}$ subunit $(\mathrm{SDHB})$ and $\mathrm{SDH} \mathrm{D}$ subunit $(S D H D)$ have been identified in $\sim 10 \%$ of $P T E N$ mutation-negative individuals with CS. ${ }^{5} S D H B$ and $S D H D$ encode the $B$ and $D$ subunits of $\mathrm{SDH}$, a Krebs-cycle enzyme that catalyzes oxidation of succinate to fumarate and also participates in the electron transport chain (complex II, succinate-ubiquinone oxidoreductase). ${ }^{5-7}$
Similar to CS, female breast cancer, papillary thyroid cancer, and renal cell carcinoma have been variably associated with individuals with germline heterozygous mutations in $S D H B$ and $S D H D .^{5-7}$ As with PTEN, the proteins associated with $S D H x$ genes also function as tumor suppressors and mutations in these genes result in mitochondrial dysfunction and tumorigenesis via upregulation of angiogenic and hypoxic pathways. ${ }^{7.8}$ Mutations in SDHA, SDHB, SDHC, SDHD, and SDH complex assembly factor 2 (SDHAF2) underlie most cases of familial paraganglioma (PGL), giving rise to PGL syndromes type 4 (PGL-4, SDHB, OMIM\# 115310), type 3 (PGL-3, SDHC, OMIM\# 605373), type 1 (PGL-1, SDHD, OMIM\# 168000), and type 2 (PGL-2, SDHAF2, OMIM\# 601650), respectively. ${ }^{9-11}$ Although PGLs and pheochromocytomas both arise from paraganglial cells, PGLs are confined to the head and neck and pheochromocytomas are localized to adrenal glands and extra-adrenal abdominal and thoracic locations. ${ }^{6}$ This is how we have defined these terms; however, we recognize that these definitions may vary depending on group or country. In addition to $S D H A, S D H B$, $S D H C, S D H D$, and SDHAF2, four additional genes are associated with the development of hereditary PGL and/or pheochromocytoma, including RET (multiple endocrine neoplasia type 2 , OMIM\# 164761), VHL (associated with von Hippel-Lindau disease, OMIM\# 193300), TMEM127 (encoding transmembrane spanning protein 127, associated with Golgi, endosomes, and

${ }^{1}$ Genomic Medicine Institute, Lerner Research Institute, Cleveland Clinic, Cleveland, Ohio, USA; ${ }^{2}$ Taussig Cancer Institute, Cleveland Clinic, Cleveland, Ohio, USA; ${ }^{3}$ Department of Genetics, Case Western Reserve University School of Medicine, Cleveland, Ohio, USA; ${ }^{4} \mathrm{CASE}$ Comprehensive Cancer Center, Case Western Reserve University School of Medicine, Cleveland, Ohio, USA. Correspondence: Charis Eng (engc@ccf.org) 
lysosomes, and hypothesized to play a role in protein trafficking and reported to negatively regulate the target of rapamycin (TOR) signaling pathway, OMIM\# 171300), and, rarely, NF1, encoding neurofibromatosis type 1 (OMIM\# 162200). ${ }^{7,12}$

In addition to their recent association with PGL in its heterozygous state, germline homozygous or compound heterozygous SDHA mutations have more commonly been associated with Leigh syndrome (OMIM\# 256000), a rare neurometabolic disorder. Succinate accumulation has been observed in SDHA mutant fibroblasts and in $S D H B$ mutant tumor tissues, ${ }^{13,14}$ and elevated urinary succinate has been associated with, but is not specific to, mitochondrial disorders, hypoxia, and seizures. Therefore, our study was designed to ascertain whether or not common biochemical alterations could be identified in individuals with CS, CS-like, and SDHx-associated pheochromocytomas and PGLs. We hypothesized that elevated succinate could be measured in urine and plasma from patients with SDHx mutations and also in individuals with PTEN mutations meeting full or partial CS diagnostic criteria. A PubMed literature search failed to identify other studies that evaluated urine and plasma succinate in patients with tumors, suggesting that ours is the first such report.

\section{MATERIALS AND METHODS}

\section{Participants}

Between October 2007 and February 2011, patients identified in the Center for Personalized Genetic Healthcare of the Genomic Medicine Institute at the Cleveland Clinic were recruited for study. Inclusion criteria included patients who met full operational diagnostic criteria for CS, partial criteria (full criteria minus one) for CS (termed CS-like), or a personal or family history of pheochromocytoma or PGL. Written informed consent was obtained from all participants. The study received ethical approval by the Cleveland Clinic Institutional Review Board for Human Subjects' Protection.

\section{Procedures}

Genomic DNA was isolated and PTEN, SDHB, SDHC, and $S D H D$ sequencing were performed based on patient phenotype using Light Scanner technology (Idaho Technology Inc., Salt Lake City, Utah). Multiplex ligation-dependent probe amplification was performed to identify PTEN, SDHB, SDHC, and $S D H D$ gene duplications or deletions in select mutation-negative individuals.

Random urine and/or blood samples were obtained during routine patient visits and de-identified. Urine and plasma were aliquoted and frozen within $1 \mathrm{~h}$ of collection and were stored at $-80^{\circ} \mathrm{C}$ until organic acid analyses were performed. Organic acid concentrations were determined using gas chromatography-mass spectrometry in the Biochemical Genetics Laboratory of ARUP, Salt Lake City, UT. This lab was blinded to the mutation status and clinical diagnosis associated with the plasma and urine samples. Measured organic acids in urine included, but were not limited to lactic acid, pyruvic acid, succinic acid, fumaric acid, 2-ketoglutaric acid, methylmalonic acid, 3-hydroxybutyric acid, acetoacetic acid, 2-keto-3-methylvaleric acid, 2-ketoisocaproic acid, 2-ketoisovaleric acid, ethylmalonic acid, adipic acid, suberic acid, sebacic acid, 4-hydroxyphenylacetic acid, 4-hydroxyphenyllactic acid, 4-hydroxy-phenylpyruvic acid, and succinylacetone. Measured organic acids in plasma included, but were not limited to, lactic acid, pyruvic acid, succinic acid, 3-hydroxybutyric acid, acetoacetic acid, 2-keto-3-methylvaleric acid, 2-ketoisocaproic acid, 2-ketoisovaleric acid, and citric acid. Reference ranges for urine and plasma organic acids were established in an age-matched population by the Biochemical Genetics Laboratory at ARUP. Urinary organic acids were reported as mmol of acid/mole of creatinine and plasma values as $\mu \mathrm{mol} / \mathrm{l}$.

\section{Statistical analysis}

Comparison of frequencies was performed with the Fisher's two-tailed exact test, with $P<0.05$ considered statistically significant.

\section{RESULTS}

A total of 66 patients (55 plasma samples; 65 urine samples) were enrolled in the study. All individuals presented to our cancer genetics clinic for an initial visit or follow-up care. Study participants whose phenotypes and family history were consistent with CS or CSL were screened for germline PTEN, SDHB, $S D H C$, and SDHD mutations. The majority of PTEN mutationnegative CS and CSL individuals were also assessed for PTEN duplications and deletions. We identified 15 PTEN mutationpositive individuals who met CS diagnostic criteria (PTEN mutation-positive), 15 PTEN mutation-negative individuals who met CS diagnostic criteria (PTEN mutation-negative, CS), 4 CSL individuals (PTEN mutation-negative, CSL), and 3 individuals with PTEN variants of unknown significance (PTEN VUS) (Table 1).

Individuals who presented with PGL, or a family history of a known $S D H$ mutation, were screened for germline $S D H B$, SDHC, SDHD, and PTEN mutations. Deletions and duplications in these genes were assessed in select mutation-negative individuals. In total, we enrolled $1 S D H B$ mutation-positive individual; 5 SDHD mutation-positive individuals; 10 individuals with PGL with no identifiable mutations, duplications, or deletions in $S D H B, S D H C$, or $S D H D$ (SDH mutation-negative, PGL), 1 individual with a known VHL mutation, and 1 individual with a known TMEM127 single-nucleotide polymorphism (Table 1).

Organic acid analyses revealed elevated plasma succinate in $13 / 21(62 \%)$ individuals with germline mutations in any examined gene as compared with 5/32 (16\%) mutation-negative controls $(P<0.001)$. The majority of PTEN mutation-positive individuals $(10 / 15 ; 67 \%)$ had elevated plasma succinate; this finding was not observed in PTEN mutation-negative CS individuals (3/15; 20\%) or the PTEN mutation-negative, CSL group (1/4; 25\%) or in individuals with PTEN variants of unknown significance $(1 / 3 ; 33 \%)$ (Table 1$)$. 
Table 1 Summary of elevated urine and plasma succinate data

$\begin{array}{lcccc}\text { Classification } & \text { Patient IDs } & \begin{array}{c}\text { Patients } \\ \text { (plasma), } \boldsymbol{n}\end{array} & \begin{array}{c}\text { Elevated plasma } \\ \text { succinate, } \boldsymbol{n}(\%)\end{array} & \begin{array}{c}\text { Elevated urine } \\ \text { Patients (urine), } \boldsymbol{n}\end{array} \\ \text { succinate, } \boldsymbol{n}(\%)\end{array}$

CSL, Cowden syndrome-like; PGL, paraganglioma; PTEN, gene encoding phosphatase and tensin homologue deleted on chromosome ten 10; SDHB, gene encoding succinate dehydrogenase B subunit; SDHD, gene encoding succinate dehydrogenase D subunit; SNP, single-nucleotide polymorphism; TMEM127, gene encoding transmembrane protein 127; VHL, gene associated with von Hippel-Lindau disease; VUS, variants of unknown significance.

Elevated plasma succinate was recorded in individuals with $\operatorname{SDHB}(1 / 1 ; 100 \%)$ and SDHD mutations $(2 / 5 ; 40 \%)$, and in one individual harboring a TMEM127 single-nucleotide polymorphism $(1 / 1 ; 100 \%)$. Elevated plasma succinate was not found in $S D H$ mutation-negative individuals with PGL (0/10; $0 \%)$ or in one individual with a mutation in $V H L(0 / 1 ; 0 \%)$ (Table 1). Elevated urine succinate was observed in some (6/19, $32 \%)$, but not all, individuals with elevated plasma succinate (Supplementary Table S1 online). No other organic acids in plasma or urine were consistently elevated or decreased for any patient group (data not shown).

\section{DISCUSSION}

This report demonstrates that elevated plasma succinate is a common finding in individuals with known pathogenic mutations in PTEN, SDHB, SDHD, and TMEM127. Previous studies have reported elevated succinate levels in tumor-derived tissue from patients with $S D H B$ mutations and in SDHA mutant fibroblasts, ${ }^{13,14}$ but to the best of our knowledge, this is the first report demonstrating an elevation of succinate in plasma from patients with germline mutations in SDHB, SDHD, TMEM127, and PTEN. Although elevated plasma succinate levels might be expected for individuals with $S D H x$ mutations, it was unexpected for PTEN mutation-positive individuals and implies that PTEN mutations somehow reduce the catalytic activity of the $\mathrm{SDH}$ protein complex.

Consistent with previous studies assessing succinate levels in tumor-derived tissue, ${ }^{14}$ more than half of our SDHD mutation-positive individuals $(3 / 5,60 \%)$ did not demonstrate elevated plasma succinate. Similarly, $33 \%$ of PTEN mutationpositive individuals did not exhibit elevated plasma succinate. Although the reason for this finding is unclear, our data suggest that elevated plasma succinate does not correlate with a specific mutation(s) or phenotype(s) (Supplementary Table S1 online). The conversion of succinate to fumarate, along with several other Krebs-cycle enzymatic reactions, is reversible. It is possible that some mutations and/or variants impair and/or enhance the reversibility of these reactions, thereby reducing succinate to normal levels. This is one possible explanation for the SDHD and PTEN mutation-positive individuals who do not exhibit elevated plasma succinate.

Elevated urine succinate was observed in some, but not all, individuals with elevated plasma succinate. The reason for this inconsistency is not entirely clear; however, it likely stems from the fact that the succinate elevations we observed in plasma samples were not severe but were mild to moderate in nature.

One of three patients harboring PTEN polymorphisms exhibited elevated succinate in both plasma and urine. This patient, unlike the other two, met full CS diagnostic criteria. It is conceivable that this intronic variant, c.210-7del5, although currently classified as a polymorphism, may actually be a pathogenic mutation leading to splicing defects.

One plausible explanation for the link between PTEN mutations and elevated plasma succinate is impaired PTEN-induced kinase 1 (PINK1), a mitochondrial localized serine-threonine kinase, transcriptionally activated by PTEN. ${ }^{15}$ Studies of PINK1 knockout mice showed impaired mitochondrial respiration in striatum, specifically; a significant decrease was seen in the state III activities for mitochondrial complex I and complex II. ${ }^{15}$ Because the oxidation of succinate to fumarate is coupled with the reduction of ubiquinone to ubiquinol, we expect that a reduction in complex II activities would simultaneously be associated with a reduction in the activity of SDH in the Krebs cycle. Therefore, mutations that affect the stability or activity of PTEN likely affect PINK1 transcription and downstream function of mitochondrial complex II.

Currently, individuals presenting to genetics clinics who meet CS diagnostic criteria or who present with familial PGL are offered the option of gene sequencing to establish the underlying cause of disease and to provide disease management. Estimated cost for PTEN sequencing, deletion, and duplication analysis is $\sim$ US $\$ 2,000$ per sample. ${ }^{16}$ Likewise, cost for $S D H B$, $S D H C$, and SDHD mutation analyses are $\sim \$ 1,000, \$ 1,300$, and $\$ 700$, respectively. Clinical multiplex ligation-dependent probe 
amplification analysis for $S D H B, S D H C$, and $S D H D$ is $\sim \$ 550$. In contrast, plasma organic acid analysis is a relatively low-cost assay. The cost of plasma organic acid analysis is $\sim \$ 230.00 \mathrm{per}$ sample. ${ }^{16}$ It is likely that the cost under contract to a medical institute would be even less. Therefore, based upon our finding that a large proportion of individuals with pathogenic PTEN, $S D H B$, and SDHD mutations exhibit elevated plasma succinate, we suggest that plasma organic acid analysis may be a useful and cost-effective preliminary screening tool for identifying individuals for whom more costly gene sequencing is warranted.

In conclusion, we have demonstrated that elevated plasma succinate is a common biochemical disturbance in the majority of PTEN, SDHB, and SDHD mutation-positive individuals and provides a plausible biochemical link for the shared phenotypic findings across these groups. Furthermore, screening for elevated succinate provides a rapid, inexpensive analytical tool for identifying individuals who are likely to harbor a germline mutation in PTEN, SDHB, or SDHD.

\section{SUPPLEMENTARY MATERIAL}

Supplementary material is linked to the online version of the paper at http://www.nature.com/gim

\section{ACKNOWLEDGMENTS}

The authors acknowledge the contributions of the Genomic Medicine Biorepository, Genomic Medicine Institute, Cleveland Clinic for preparation of genomic DNA and processing of blood and urine samples, and Yiran Yang, Jin Lian Chen, and Ying Ni (all of the Eng lab) and the Genomics Core Facility, Genomic Medicine Institute, Cleveland Clinic for PCR-based Sanger sequencing and multiplex ligation-dependent probe amplification analyses. C.E. is the Sondra J. and Stephen R. Hardis Chair of Cancer Genomic Medicine at the Cleveland Clinic and holds an American Cancer Society Clinical Research Professorship, generously funded, in part, by the F.M. Kirby Foundation. This study was funded by the Breast Cancer Research Foundation.

\section{DISCLOSURE}

The authors declare no conflict of interest.

\section{REFERENCES}

1. Hobert JA, Eng C. PTEN hamartoma tumor syndrome: an overview. Genet Med 2009;11:687-694.

2. Tan MH, Mester J, Peterson C, et al. A clinical scoring system for selection of patients for PTEN mutation testing is proposed on the basis of a prospective study of 3042 probands. Am J Hum Genet 2011;88:42-56.

3. Ngeow J, Mester J, Rybicki LA, Ni Y, Milas M, Eng C. Incidence and Clinical Characteristics of Thyroid Cancer in Prospective Series of Individuals with Cowden and Cowden-Like Syndrome Characterized by Germline PTEN, SDH, or KLLN Alterations. J Clin Endocrinol Metab, 2011;96:E2063-E2071.

4. Hollander MC, Blumenthal GM, Dennis PA. PTEN loss in the continuum of common cancers, rare syndromes and mouse models. Nat Rev Cancer 2011;11:289-301.

5. Ni Y, Zbuk KM, Sadler T, et al. Germline mutations and variants in the succinate dehydrogenase genes in Cowden and Cowden-like syndromes. Am J Hum Genet 2008;83:261-268.

6. Neumann HP, Pawlu C, Peczkowska M, et al.; European-American Paraganglioma Study Group. Distinct clinical features of paraganglioma syndromes associated with SDHB and SDHD gene mutations. JAMA 2004;292:943-951.

7. Timmers HJ, Gimenez-Roqueplo AP, Mannelli M, Pacak K. Clinical aspects of SDHx-related pheochromocytoma and paraganglioma. Endocr Relat Cancer 2009;16:391-400.

8. Gottlieb E, Tomlinson IP. Mitochondrial tumour suppressors: a genetic and biochemical update. Nat Rev Cancer 2005;5:857-866.

9. Burnichon N, Brière JJ, Libé R, et al. SDHA is a tumor suppressor gene causing paraganglioma. Hum Mol Genet 2010;19:3011-3020.

10. Young WF Jr, Abboud AL. Editorial: paraganglioma-all in the family. J Clin Endocrinol Metab 2006;91:790-792.

11. Kunst HP, Rutten MH, de Mönnink JP, et al. SDHAF2 (PGL2-SDH5) and hereditary head and neck paraganglioma. Clin Cancer Res 2011;17:247-254.

12. Qin $Y$, Yao L, King EE, et al. Germline mutations in TMEM127 confer susceptibility to pheochromocytoma. Nat Genet 2010;42:229-233.

13. Brière JJ, Favier J, Bénit $P$, et al. Mitochondrial succinate is instrumental for HIF1alpha nuclear translocation in SDHA-mutant fibroblasts under normoxic conditions. Hum Mol Genet 2005;14:3263-3269.

14. Pollard PJ, Brière JJ, Alam NA, et al. Accumulation of Krebs cycle intermediates and over-expression of HIF1alpha in tumours which result from germline $\mathrm{FH}$ and SDH mutations. Hum Mol Genet 2005;14:2231-2239.

15. Gautier CA, Kitada T, Shen J. Loss of PINK1 causes mitochondrial functional defects and increased sensitivity to oxidative stress. Proc Natl Acad Sci USA 2008;105:11364-11369.

16. Greenwood Genetic Center. Tests \& costs. http://www.ggc.org/diagnostic/testcosts.html 